\title{
Oral Sodium Phenylbutyrate
}

National Cancer Institute

\section{Source}

National Cancer Institute. Oral Sodium Phenylbutyrate. NCI Thesaurus. Code C67083.

An orally active derivative of the short-chain fatty acid butyrate with potential

antineoplastic activity. 4-Phenylbutyrate inhibits histone deacetylase, resulting in cell cycle gene expression modulation, reduced cell proliferation, increased cell differentiation, and apoptosis. This agent also initiates fragmentation of genomic DNA, resulting in decreased DNA synthesis and the inhibition of tumor cell migration and invasion. 